\title{
Large scale exfoliation of inorganic layered compounds in aqueous surfactant solutions
}

Ronan J Smith ${ }^{1}$, Paul J King ${ }^{1}$, Mustafa Lotya ${ }^{1}$, Christian Wirtz ${ }^{1}$, Umar Khan ${ }^{1}$, Sukanta De ${ }^{1}$, Arlene O’Neill ${ }^{1}$, Georg S Duesberg, ${ }^{2}$ Jaime C Grunlan $^{3}$, Gregory Moriarty ${ }^{3}$, Jun Chen ${ }^{4}$, Jiazhao Wang ${ }^{5}$, Andrew Minett ${ }^{6}$, Valeria Nicolosi ${ }^{7}$ and Jonathan N Coleman ${ }^{1 *}$

${ }^{1}$ School of Physics and CRANN, Trinity College Dublin, Dublin 2, Ireland

${ }^{2}$ School of Chemistry and CRANN, Trinity College Dublin, Dublin 2, Ireland

${ }^{3}$ Department of Mechanical Engineering, Texas A\&M University, College Station, Texas 77843, USA

${ }^{4}$ Intelligent Polymer Research Institute, ARC Centre of Excellence for Electromaterials Science, AIIM Facility, University of Wollongong, NSW 2522, Australia

${ }^{5}$ Institute for Superconducting and Electronic Materials, ARC Centre of Excellence for Electromaterials Science, University of Wollongong, NSW 2522, Australia

${ }^{6}$ Laboratory for Sustainable Technology, School of Chemical and Biomolecular Engineering, University of Sydney, Sydney, NSW 2006, Australia.

${ }^{7}$ Department of Materials, University of Oxford, Parks Road, Oxford OX1 3PH, UK *colemaj@tcd.ie

Keywords

Nanosheet, exfoliation, surfactant, thermoelectric, battery

Table of contents text:

We have developed methods to exfoliate $\mathrm{MoS}_{2}$ in large quantities in surfactant-water solutions. This method can be extended to a range of other layered compounds. The layered material tends to be exfoliated as relatively defect free flakes with lateral sizes of $100 \mathrm{~s}$ of $\mathrm{nm}$. 
With high surface area and novel properties, two-dimensional (2D) materials are potentially useful for a range of applications. In addition to graphene, many $2 \mathrm{D}$ compounds exist with $\mathrm{BN}, \mathrm{MoS}_{2}$ and $\mathrm{Bi}_{2} \mathrm{Te}_{3}$ generating renewed interest. Such materials are found stacked in layered crystals and can be metals, semiconductors or insulators. ${ }^{[1]}$ In addition, some are thermoelectric materials, ${ }^{[2]}$ superconductors ${ }^{[3]}$ or topological insulators ${ }^{[4]}$. In order to unlock these valuable properties, these materials must be easily exfoliated to individual layers. Here we show a simple, large-scale method to exfoliate $\mathrm{BN}$, transition metal dichalcogenides and transition metal oxides down to very thin platelets in water. The platelets are stabilised against re-aggregation by a surfactant coating. Liquid phase exfoliation allows the formation of films

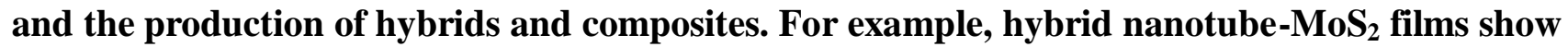
promise for thermoelectric and battery applications while $\mathrm{NbSe}_{2}$ films show potential as transparent conductors.

In addition to graphite, a wide range of inorganic layered compounds exist. A good example are the transition metal dichalcogenides (TMDs). ${ }^{[1]}$ These form 2-dimensional layers of covalently bonded transition metal atoms, $M$, and chalcogen atoms, $X(X=S, S e, T e)$, in the stoichiometry $\mathrm{MX}_{2}$, with examples such as $\mathrm{MoS}_{2}, \mathrm{NbSe}_{2}$ and $\mathrm{NiTe}_{2} \cdot{ }^{\left[{ }^{[1]}\right.}$ In addition, some transition metal oxides ${ }^{[5]}$ form $2 \mathrm{D}$ structures as do other materials such as $\mathrm{BN}, \mathrm{Bi}_{2} \mathrm{Te}_{3}, \mathrm{Sb}_{2} \mathrm{Se}_{3}$, FeTe etc. In general these 2D layers tend to bond via van der Waals interactions, stacking to form 3D crystals. These materials span the whole gamut of electronic structures from insulator to metal ${ }^{[1]}$ and display interesting properties ${ }^{[6]}$ such as superconductivity, ${ }^{[3]}$ thermoelectricity ${ }^{[2]}$ and topological insulator effects. ${ }^{[4]}$

While micro-mechanically exfoliated ${ }^{[7]}$ single flakes of materials such as $\mathrm{MoS}_{2}$ are ideal for electronic devices, ${ }^{[8]}$ large scale liquid-phase exfoliation methods will lead to a range of thin film applications such as nano-scale hybrids for use in thermoelectrics, ${ }^{[9]}$ supercapacitors ${ }^{[10]}$ or Li-ion batteries $^{[11]}$. One advantage of such applications is that, as the electronic properties of TMDs vary relatively slowly with layer number, ${ }^{[12,13]}$ full exfoliation to monolayers is not necessary; dispersed few-layer flakes are sufficient.

While a number of layered compounds can be exfoliated by ion intercalation, ${ }^{[14-17]}$ this method is time consuming, extremely sensitive to environmental conditions and results in structural deformations in some TMDs. ${ }^{[18]}$ Furthermore, removal of the ions results in re-aggregation of the layers. ${ }^{[19]}$ More promisingly, it has recently been shown that both TMDs ${ }^{[20]}$ and BN can be exfoliated in organic solvents. ${ }^{[21-24]}$ However, for large-scale applications, exfoliation in an aqueous environment would be hugely advantageous. While $\mathrm{BN}$ can be dispersed in water due to sonication-assisted hydrolysis, this method cannot be extended to other layered compounds. ${ }^{[25]}$ The discovery of a facile, scalable method to exfoliate a range of layered materials in water would assist the production and 
characterisation of a range of new materials and greatly facilitate the potential transfer of such technology to industry. In this work we show that a number of layered crystals can be exfoliated in water, resulting in thin flakes stabilised by a surfactant coating. This method is robust, can be carried out in ambient conditions, is scalable and allows the preparation of films, hybrids and composites.

One possible reason why ion intercalation has been prevalent for TMDs rather than other liquid based dispersion methods is the relatively high exfoliation (surface) energy of TMDs. Computational

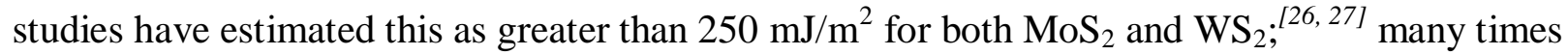
greater than that of graphene ${ }^{[28]}$ or $\mathrm{BN}^{[29]}$. We suggest that sonication can be used to exfoliate TMDs in water, so long as a stabiliser is present to prevent rapid reaggregation driven by the large surface energy. ${ }^{[14]}$ We suggest that ionic surfactants are ideal stabilisers due to their van der Waals binding to the exfoliated nanosheets and subsequent electrostatic stabilisation.

To test this, we probe sonicated $\mathrm{MoS}_{2}$ powder at initial concentration, $\mathrm{C}_{\mathrm{I}}=5 \mathrm{mg} / \mathrm{mL}$, in an aqueous solution of the surfactant sodium cholate ${ }^{[30,31]}$ of concentration, $\mathrm{C}_{\mathrm{SC}}=1.5 \mathrm{mg} / \mathrm{mL}$ for 30 minutes (although other surfactants work just as well, see supporting information, SI). The dispersions were centrifuged to remove un-exfoliated powder. Separation of the supernatant gave dark liquids as in figure 1a. Optical absorption measurements gave spectra superimposed on a large power-law background, $\sim \lambda^{-a}$ (figure $1 \mathrm{~b}$ and SI). The exponent, a, associated with this background varied with centrifugation rate, and so flake size, suggesting the background to be due to light scattering . ${ }^{[32]}$ Subtraction of the scattering background gave spectra typical of $\mathrm{MoS}_{2}$ (figure 1b, inset). ${ }^{[33]}$ Optical absorbance scaled linearly with C (see SI) both before and after background subtraction.

The dispersed concentration, $\mathrm{C}$, could be measured by filtration and weighing allowing the estimation of the extinction coefficient (672 nm, after background subtraction) to be $\alpha=1517 \mathrm{~L} / \mathrm{g} / \mathrm{m}$. This allows the measurement of dispersed concentrations using the Lambert-Beer law, $A / l=\alpha C$ where $\mathrm{A} / \mathrm{l}$ is the absorbance per cell length. The dispersed concentration increased monotonically with surfactant concentration as shown in figure 1c. Interestingly, reasonable concentrations of $\mathrm{MoS}_{2}$ could be dispersed even at surfactant concentrations well below the critical micelle concentration ( $\sim 4 \mathrm{mg} / \mathrm{mL}$ ) of sodium cholate, as long as $\mathrm{C}_{\mathrm{SC}} \geq 0.3 \mathrm{C}_{\mathrm{I}}$. In addition, the dispersed concentration scales linearly with the starting concentration $\left(C_{I}\right)$ once $C_{I}<20 \mathrm{mg} / \mathrm{mL}$. This allows us to define a yield, $\mathrm{Y}\left(\mathrm{C}=\mathrm{YC}_{\mathrm{I}}\right)$. By increasing the sonication time to $16 \mathrm{hrs}$, the dispersed concentration can be increased to $\sim 0.5 \mathrm{mg} / \mathrm{mL}$. This is equivalent to a yield of $\sim 10 \%$. By recycling the sediment, the overall yield can be increased to $17 \%$ (see SI). We suggest that multiple recycling steps could increase the yield even further. We note that this method can easily be used to make dispersions by the litre with concentrations of at least $0.25 \mathrm{mg} / \mathrm{ml}$ (see SI). 
Surfactant-coated nanoparticles are usually stabilised by electrostatic repulsion as characterised by the zeta potential. ${ }^{[34,35]}$ At neutral $\mathrm{pH}$, the zeta potential of the sodium cholate-coated $\mathrm{MoS}_{2}$ was measured to be stable over weeks at $-40 \mathrm{mV}$ (figure 1f) and was extremely robust against changes in $\mathrm{pH}$ (figure 1f). After centrifugation, sedimentation measurements ${ }^{[36]}$ showed that over half the $\mathrm{MoS}_{2}$ remains dispersed indefinitely (figure $1 \mathrm{~g}$ ). We note that the observation of such stable dispersions at relatively low zeta potential suggests that previous estimates of $\mathrm{MoS}_{2}$ surface energy may be too high. ${ }^{[26,27]}$

It is critical to ascertain the nature of the dispersed $\mathrm{MoS}_{2}$. Transmission electron microscope examination of material deposited from the dispersion showed the presence of large quantities of extremely thin 2-dimensional flakes (figures $2 \mathrm{a}-\mathrm{c}$ ) which we believe to consist of a small number of stacked $\mathrm{MoS}_{2}$ monolayers (see SI for a library of TEM images). The diffraction pattern in figure 2a is consistent with an undistorted lattice. High resolution TEM and scanning TEM show that individual layer edges can be observed near the periphery of these flakes (figure $2 \mathrm{~d}-\mathrm{h}$ ). By counting these edges, we estimate exfoliated flakes to contain 2 to 9 layers (see SI). The high resolution images in $\mathrm{f}, \mathrm{i}$ $\& \mathrm{j}$ show clean, well defined, hexagonally symmetric structures. In a typical optimised dispersion, the mean flake length is $280 \mathrm{~nm}$. However, selection of the larger flakes by column chromatography can increase this to at least $430 \mathrm{~nm}$ (see SI).

Exfoliation of layered materials in an aqueous environment can be considered a gateway technology as it allows a wide range of processing techniques to be applied. Once a stable, environmentally robust dispersion has been prepared, it is straightforward to prepare films by vacuum filtration with thickness from $\sim \mathrm{nm}$ to $10 \mathrm{~s}$ of $\mu \mathrm{m}$. SEM imaging of a thin $\mathrm{MoS}_{2}$ film shows a disordered array of $\mathrm{MoS}_{2}$ flakes stacked predominately in-plane (figure 3a). Absorption spectroscopy shows that $\mathrm{MoS}_{2}$ films are semiconducting in nature with an optical gap of $\sim 1.6 \mathrm{eV}$ (figure $3 \mathrm{~b}$ ). Raman spectroscopy shows intense peaks at positions almost identical to those observed in $\mathrm{MoS}_{2}$ powder $\left(\sim 375\right.$ and $\left.405 \mathrm{~cm}^{-1}\right)$ which, taken with the electron diffraction results, strongly suggesting the structure to be undistorted $2 \mathrm{H} \mathrm{MoS}_{2}$ (figure $3 \mathrm{c}$ ). ${ }^{[20]}$ This is in contrast to ion exfoliated $\mathrm{MoS}_{2}$ which generally becomes distorted from the bulk $2 \mathrm{H}$ structure. ${ }^{[18,37]}$

TMDs would be ideal for applications in thermoelectric devices, $\mathrm{Li}$ ion batteries or supercapacitors but for their low electronic conductivity. However, the conductivity would be increased dramatically by incorporation of conducting nanostructures into the TMD films. To demonstrate this, we exfoliated graphene and single walled nanotubes (SWNT) in aqueous sodium cholate solutions at known concentrations. ${ }^{[31]}$ These were then blended with an aqueous $\mathrm{MoS}_{2} / \mathrm{SC}$ dispersion in various ratios to give $\mathrm{MoS}_{2} /$ graphene and $\mathrm{MoS}_{2} / \mathrm{SWNT}$ dispersions with a range of compositions. These could then be formed into free standing films by vacuum filtration (figure $3 \mathrm{~d}$ ). 
SEM analysis shows the $\mathrm{MoS}_{2}$ /graphene films to be similar in morphology to the $\mathrm{MoS}_{2}$-only films while for the $\mathrm{MoS}_{2} / \mathrm{SWNT}$ films, the flakes appear to be embedded in the SWNT network (figure 3f). Addition of the nano-conductors increases the film conductivity, $\sigma_{\mathrm{DC}}$, dramatically from $\sim 10^{-5} \mathrm{~S} / \mathrm{m}$ for the $\mathrm{MoS}_{2}$ alone to $1000 \mathrm{~S} / \mathrm{m}$ for $100 \%$ graphene and $2 \times 10^{5} \mathrm{~S} / \mathrm{m}$ for $75 \%$ SWNTs (figure $3 \mathrm{f}$ ).

Increasing the DC conductivity of nanostructured materials without degrading the Seebeck coefficient is an important goal in thermoelectric research. ${ }^{[9]}$ We found the Seebeck coefficient to fall only slightly with nanotube content, remaining close to $\mathrm{S}=25 \mu \mathrm{V} / \mathrm{K}$ up to $75 \mathrm{wt} \%$ SWNT. Importantly, the power factor increased with nanotube content (figure $3 \mathrm{~g}$ ), reaching $\mathrm{S}^{2} \sigma_{\mathrm{DC}}=87 \mu \mathrm{Wm}^{-1} \mathrm{~K}^{-2}$ for $75 \mathrm{wt} \%$ before falling off at higher nanotube contents.

We can also use $\mathrm{MoS}_{2} / \mathrm{SWNT}$ hybrids as cathodes in Li ion batteries. These hybrid electrodes show higher rate capability and higher retained capacity over 100 cycles when compared to $\mathrm{MoS}_{2}$-only electrodes (figure 3h). The very high Coulombic efficiency (above 95\%) of the $\mathrm{MoS}_{2}-\mathrm{CNT}$ hybrid electrode suggests very good electrochemical performance as confirmed by ac impedance spectroscopy (see SI).

This method is not limited to $\mathrm{MoS}_{2}$ but can be extended to a wide range of layered compounds such as $\mathrm{BN}, \mathrm{WS}_{2}, \mathrm{TaSe}_{2}, \mathrm{MoTe}_{2}, \mathrm{MoSe}_{2}$ and $\mathrm{NbSe}_{2}$. For these materials, stable dispersions were prepared, even though the dispersion procedure was not optimised for each material (figure $4 \mathrm{a}$ ). The absorption spectra of the dispersions were close to those expected for these materials (figure $4 \mathrm{~b}) .{ }^{[1]}$ In addition, these dispersions could easily be formed into films by filtration (figure 4c). TEM examination showed reasonably well-exfoliated flakes in all cases (figure $4 d-i$ ). We illustrate the usefulness of this method by making electrical and optical measurements on an $\mathrm{NbSe}_{2}$ film (thickness $\sim 200 \mathrm{~nm})$. We measure transmittance $(550 \mathrm{~nm})$ of $\mathrm{T}=20 \%$ coupled with sheet resistance of $\mathrm{R}_{\mathrm{s}}=2.1$ $\mathrm{k} \Omega / \square$. By adding $10 \mathrm{wt} \%$ SWNTs, these properties improved to $\mathrm{T}=33 \%$ and $\mathrm{R}_{\mathrm{s}}=67 \Omega / \square$, significantly better than for graphene networks (see SI). ${ }^{[38]}$ This exfoliation method can also be extended to transition metal oxides. We have exfoliated flakes of $\mathrm{MnO}_{2}$ (figure $4 \mathrm{j} \& \mathrm{k}$ ), emphasising the generality of this method. Such materials will be important in applications such as supercapacitors. ${ }^{[39,40]}$

We propose that this exfoliation method is general and will apply to the vast majority of layered compounds. Unlike ion exfoliation methods, this process is quick, easy and insensitive to ambient conditions. While the degree of exfoliation is inferior to ion exfoliated dispersions, this is more than compensated by the versatility of the procedure (see SI). This method will lead to the ability to produce thin films of inorganic layered compounds for applications in batteries and devices or as coatings or lubricants. In addition, it allows the production of a wide range of hybrids with tuneable 
conductivity and attractive thermoelectric properties as well as composites with enhanced mechanical properties.

Methods

Dispersions were typically made by probe sonicating (Sonics VX-750) layered compounds (typically $\mathrm{C}_{\mathrm{I}}=5 \mathrm{mg} / \mathrm{ml}$ ) in powder form in an aqueous surfactant solution (typically $\mathrm{C}_{\mathrm{SC}}=1.5 \mathrm{mg} / \mathrm{ml}$ ) for 30 minutes. The dispersions were then centrifuged (Hettich Mickro 22R) for 90 minutes, typically at $1500 \mathrm{rpm}$. Absorption spectra were obtained with a Varian Cary 6000i while zeta potential was measured with a Malvern Zetasizer Nano. TEM was performed with a Jeol 2100 operated at $200 \mathrm{kV}$, HRTEM and low resolution STEM using an FEI Titan and high resolution STEM using a Jeol 2200MCO FEGTEM/STEM, fitted with two CEOS Cs aberration correctors, operated at 200kV. Microscopy samples were prepared by drop casting onto a holey carbon grid. Raman spectroscopy was carried out on a Horiba Jobin Yvon LabRAM using a 50X objective lens with a $633 \mathrm{~nm}$ laser excitation. SEM was carried out using a Zeiss Ultra Plus SEM. More detail is given in the supporting information.

\section{Acknowledgements}

The authors thank Science Foundation Ireland for financial support through the Principle Investigator scheme, grant number 07/IN.1/I1772, the Research Frontiers Program; grant number 09/RFP/MTR2286 and the European Research Council (grant - SEMANTICS). ML and AO’N acknowledge IRCSET for support. JCG and GM acknowledge financial support from the US Air Force Office of Scientific Research (Grant No. FA9550-09-1-0609), under the auspices of Dr. Charles Lee, and the Texas Engineering Experiment Station (TEES). 
[1] J. A. Wilson, A. D. Yoffe, Advances in Physics 1969, 18, 193.

[2] X. F. Tang, W. J. Xie, H. Li, W. Y. Zhao, Q. J. Zhang, M. Niino, Applied Physics Letters 2007, 90.

[3] F. R. Gamble, B. G. Silbernagel, Journal of Chemical Physics 1975, 63, 2544.

[4] H. J. Zhang, C. X. Liu, X. L. Qi, X. Dai, Z. Fang, S. C. Zhang, Nature Physics 2009, 5, 438.

[5] M. Osada, T. Sasaki, Journal of Materials Chemistry 2009, 19, 2503.

[6] R. H. Friend, A. D. Yoffe, Advances in Physics 1987, 36, 1.

[7] K. S. Novoselov, D. Jiang, F. Schedin, T. J. Booth, V. V. Khotkevich, S. V. Morozov, A. K. Geim, Proceedings of the National Academy of Sciences of the United States of America 2005, 102, 10451.

[8] B. Radisavljevic, A. Radenovic, J. Brivio, V. Giacometti, A. Kis, Nat Nano 2011, 6, 147.

[9] M. S. Dresselhaus, G. Chen, M. Y. Tang, R. G. Yang, H. Lee, D. Z. Wang, Z. F. Ren, J. P. Fleurial, P. Gogna, Advanced Materials 2007, 19, 1043.

[10] J. M. Soon, K. P. Loh, Electrochem. Solid State Lett. 2007, 10, A250.

[11] C. Q. Feng, J. Ma, H. Li, R. Zeng, Z. P. Guo, H. K. Liu, Mater. Res. Bull. 2009, 44, 1811.

[12] K. F. Mak, C. Lee, J. Hone, J. Shan, T. F. Heinz, Phys. Rev. Lett. 2010, 105, 4.

[13] A. Splendiani, L. Sun, Y. B. Zhang, T. S. Li, J. Kim, C. Y. Chim, G. Galli, F. Wang, Nano Letters 2010, 10, 1271.

[14] P. Joensen, R. F. Frindt, S. R. Morrison, Mater. Res. Bull. 1986, $21,457$.

[15] D. Yang, R. F. Frindt, Journal of Physics and Chemistry of Solids 1996, 57, 1113.

[16] H. Matte, A. Gomathi, A. K. Manna, D. J. Late, R. Datta, S. K. Pati, C. N. R. Rao, Angewandte Chemie-International Edition 2010, 49, 4059.

[17] Z. F. Ding, L. Viculis, J. Nakawatase, R. B. Kaner, Advanced Materials 2001, 13, 797.

[18] S. J. Sandoval, D. Yang, R. F. Frindt, J. C. Irwin, Physical Review B 1991, 44, 3955.

[19] R. Bissessur, J. Heising, W. Hirpo, M. Kanatzidis, Chemistry of Materials 1996, 8, 318.

[20] J. N. Coleman, M. Lotya, A. O'Neill, S. D. Bergin, P. J. King, U. Khan, K. Young, A. Gaucher, S. De, R. J. Smith, I. V. Shvets, S. K. Arora, G. Stanton, H. Y. Kim, K. Lee, G. T. Kim, G. S. Duesberg, T. Hallam, J. J. Boland, J. J. Wang, J. F. Donegan, J. C. Grunlan, G. Moriarty, A. Shmeliov, R. J. Nicholls, J. M. Perkins, E. M. Grieveson, K. Theuwissen, D. W. McComb, P. D. Nellist, V. Nicolosi, Science 2011, 331, 568.

[21] W. Q. Han, L. J. Wu, Y. M. Zhu, K. Watanabe, T. Taniguchi, Applied Physics Letters 2008, 93.

[22] Y. Lin, T. V. Williams, J. W. Connell, Journal of Physical Chemistry Letters 2010, 1, 277.

[23] J. H. Warner, M. H. Rummeli, A. Bachmatiuk, B. Buchner, Acs Nano 2010, 4, 1299. 
[24] C. Y. Zhi, Y. Bando, C. C. Tang, H. Kuwahara, D. Golberg, Advanced Materials 2009, 21, 2889.

[25] Y. Lin, T. V. Williams, T. B. Xu, W. Cao, H. E. Elsayed-Ali, J. W. Connell, Journal of Physical Chemistry C 2011, 115, 2679.

[26] J. D. Fuhr, J. O. Sofo, A. Saul, Physical Review B 1999, 60, 8343.

[27] K. Weiss, J. M. Phillips, Physical Review B 1976, 14, 5392.

[28] Y. Hernandez, V. Nicolosi, M. Lotya, F. M. Blighe, Z. Y. Sun, S. De, I. T. McGovern, B. Holland, M. Byrne, Y. K. Gun'ko, J. J. Boland, P. Niraj, G. Duesberg, S. Krishnamurthy, R. Goodhue, J. Hutchison, V. Scardaci, A. C. Ferrari, J. N. Coleman, Nature Nanotechnology 2008, 3, 563.

[29] N. Rathod, S. G. Hatzikiriakos, Polymer Engineering and Science 2004, 44, 1543.

[30] T. Hertel, A. Hagen, V. Talalaev, K. Arnold, F. Hennrich, M. Kappes, S. Rosenthal, J. McBride, H. Ulbricht, E. Flahaut, Nano Letters 2005, 5, 511.

[31] M. Lotya, P. J. King, U. Khan, S. De, J. N. Coleman, Acs Nano 2010, 4, 3155.

[32] C. F. Bohren, D. R. Huffman, Absorption and Scattering of Light by Small Particles, Wiley, New York 1998.

[33] J. P. Wilcoxon, P. P. Newcomer, G. A. Samara, Journal of Applied Physics 1997, 81, 7934.

[34] M. Lotya, Y. Hernandez, P. J. King, R. J. Smith, V. Nicolosi, L. S. Karlsson, F. M. Blighe, S. De, Z. M. Wang, I. T. McGovern, G. S. Duesberg, J. N. Coleman, Journal of the American Chemical Society 2009, 131, 3611.

[35] Z. Sun, V. Nicolosi, D. Rickard, S. D. Bergin, D. Aherne, J. N. Coleman, Journal of Physical Chemistry C 2008, 112, 10692.

[36] V. Nicolosi, D. Vrbanic, A. Mrzel, J. McCauley, S. O'Flaherty, C. McGuinness, G. Compagnini, D. Mihailovic, W. J. Blau, J. N. Coleman, Journal of Physical Chemistry B 2005, 109, 7124.

[37] G. L. Frey, K. J. Reynolds, R. H. Friend, H. Cohen, Y. Feldman, Journal of the American Chemical Society 2003, 125, 5998.

[38] S. De, J. N. Coleman, Acs Nano 2010, 4, 2713.

[39] P. C. Chen, G. Z. Shen, Y. Shi, H. T. Chen, C. W. Zhou, Acs Nano 2010, 4, 4403.

[40] S. W. Lee, J. Kim, S. Chen, P. T. Hammond, Y. Shao-Horn, Acs Nano, 4, 3889. 

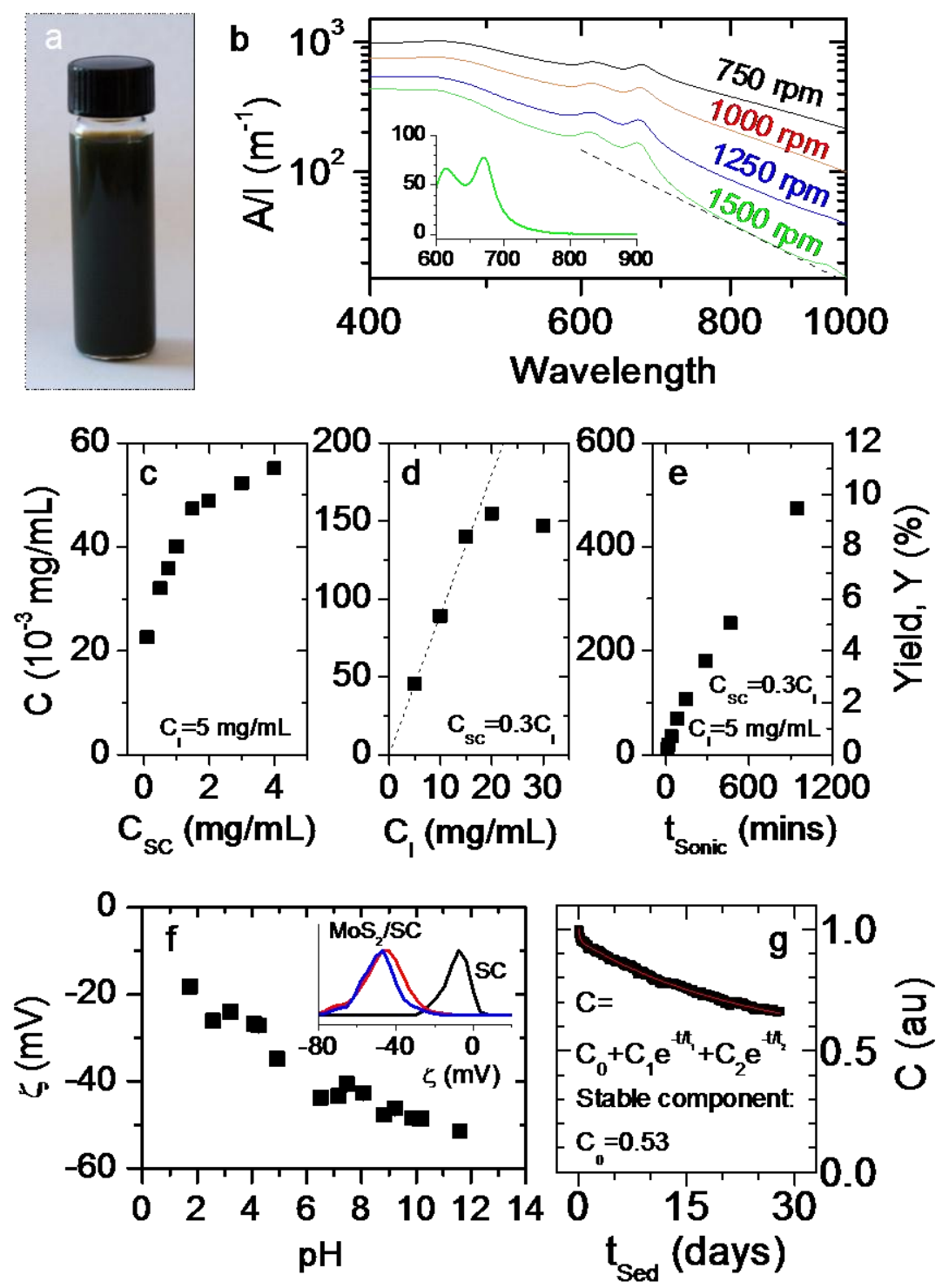

Figure 1: Exfoliation of $\mathrm{MoS}_{2}$. a, A dispersion of $\mathrm{MoS}_{2}$ flakes in water, stabilised by sodium cholate (SC). b, Absorption spectra of $\mathrm{MoS}_{2} / \mathrm{SC}$ prepared by centrifuging at different rates. Inset: The spectrum of the $1500 \mathrm{rpm}$ dispersion after subtraction of scattering background (dashed line in main figure). $\mathrm{MoS}_{2}$ concentration after centrifugation $(1500 \mathrm{rpm})$ as a function of $\mathrm{c}, \mathrm{SC}$ concentration $\left(\mathrm{C}_{\mathrm{SC}}\right)$, $\mathrm{d}$, initial $\mathrm{MoS}_{2}$ concentration $\left(\mathrm{C}_{\mathrm{I}}\right)$ and e, sonication time. f, Inset: Zeta potential spectra for SC solution and $\mathrm{MoS}_{2} / \mathrm{SC}$ dispersions immediately after preparation (dashed) and after 1 week (solid). Main figure: Zeta potential of $\mathrm{MoS}_{2} / \mathrm{SC}$ dispersions as a function of $\mathrm{pH}$ (adjusted by addition of $1 \mathrm{M} \mathrm{HCl}$ and $\mathrm{NaOH})$. g, Concentration remaining as a function of sedimentation time for an $\mathrm{MoS}_{2} / \mathrm{SC}$ dispersion over the course of one month. The data has been fitted to sedimentation theory for two sedimenting components ( 1 and 2 ) and a stable component $(0)$. The fit constants are $\mathrm{C}_{0}=0.53, \mathrm{C}_{1}=0.44, \mathrm{C}_{2}=0.05$, 
$\mathrm{t}_{1}=24$ days, $\mathrm{t}_{2}=5.5 \mathrm{hrs}$. The sedimentation components probably represent large flakes ( 1$)$ and residual undispersed powder (2).
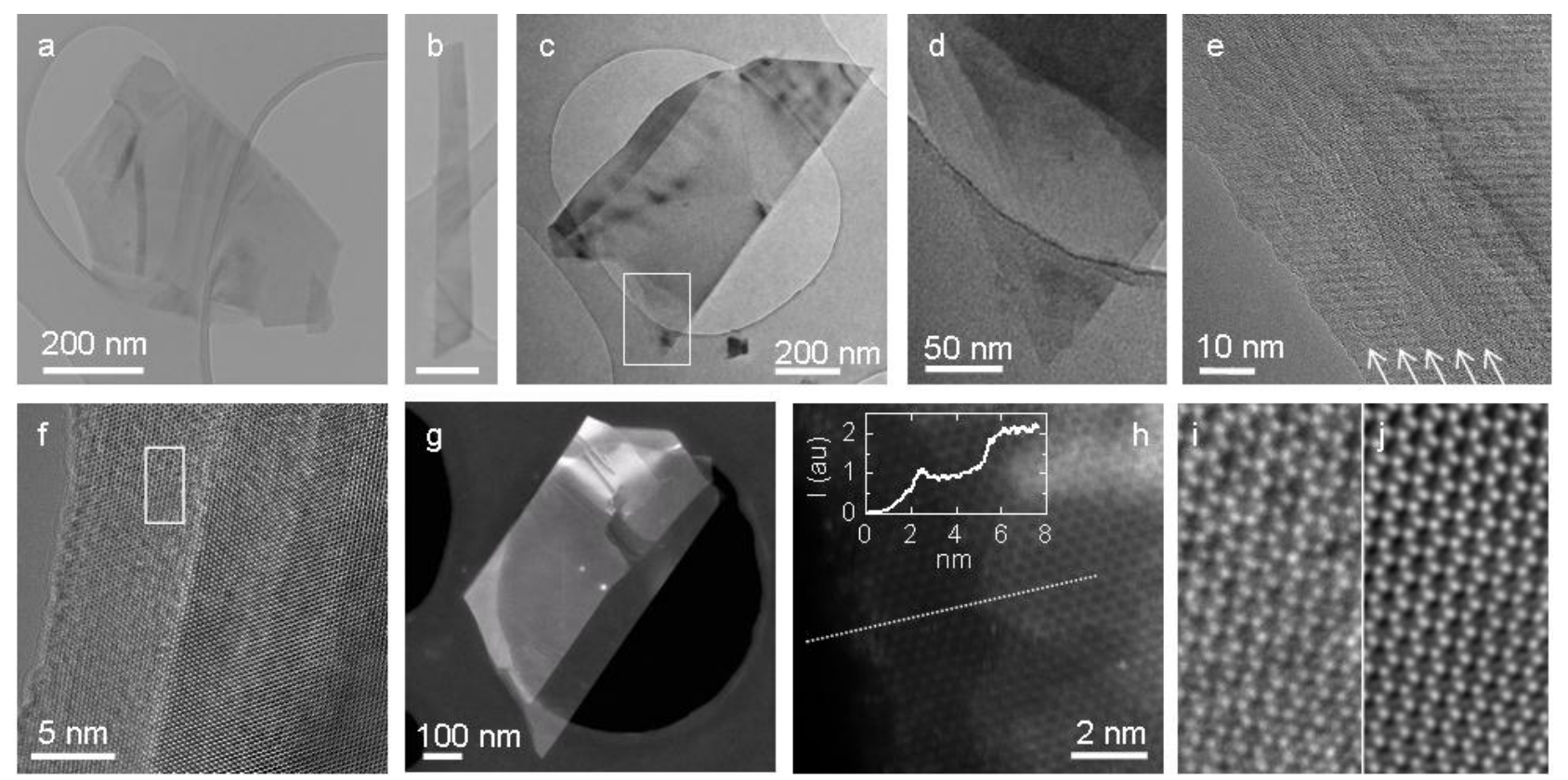

Figure 2: Transmission electron microscopy of $\mathrm{MoS}_{2}$ flakes. a , b \& c Typical bright field TEM images of $\mathrm{MoS}_{2}$ flakes. A inset Typical electron diffraction pattern. $\mathrm{d}$, A zoomed bright field image of the region in $\mathrm{c}$ indicated by the white square showing layer edges. e \& $\mathrm{f}$, Phase contrast HRTEM images of a thin $\mathrm{MoS}_{2}$ flakes showing layer edges. g, Dark field scanning TEM image of a thin flake clearly illustrate the step edges. h, An unfiltered aberration corrected high angle annular dark field (HAADF) STEM. The HAADF STEM detector was calibrated in units of primary electrons detected. This has enabled quantitative interpretation of Z-contrast STEM images and the counting statistics of the signal. Inset: Average signal intensity plotted over the dashed line. i, A zoomed image of the region in $\mathrm{f}$ indicated by the white square showing the $\mathrm{MoS}_{2}$ atomic structure. $\mathrm{j}$, A digitally filtered (low-pass FFT with spot masking and a 25 point smoothing) image of the same region as in g clearly showing defect free hexagonal symmetry. 

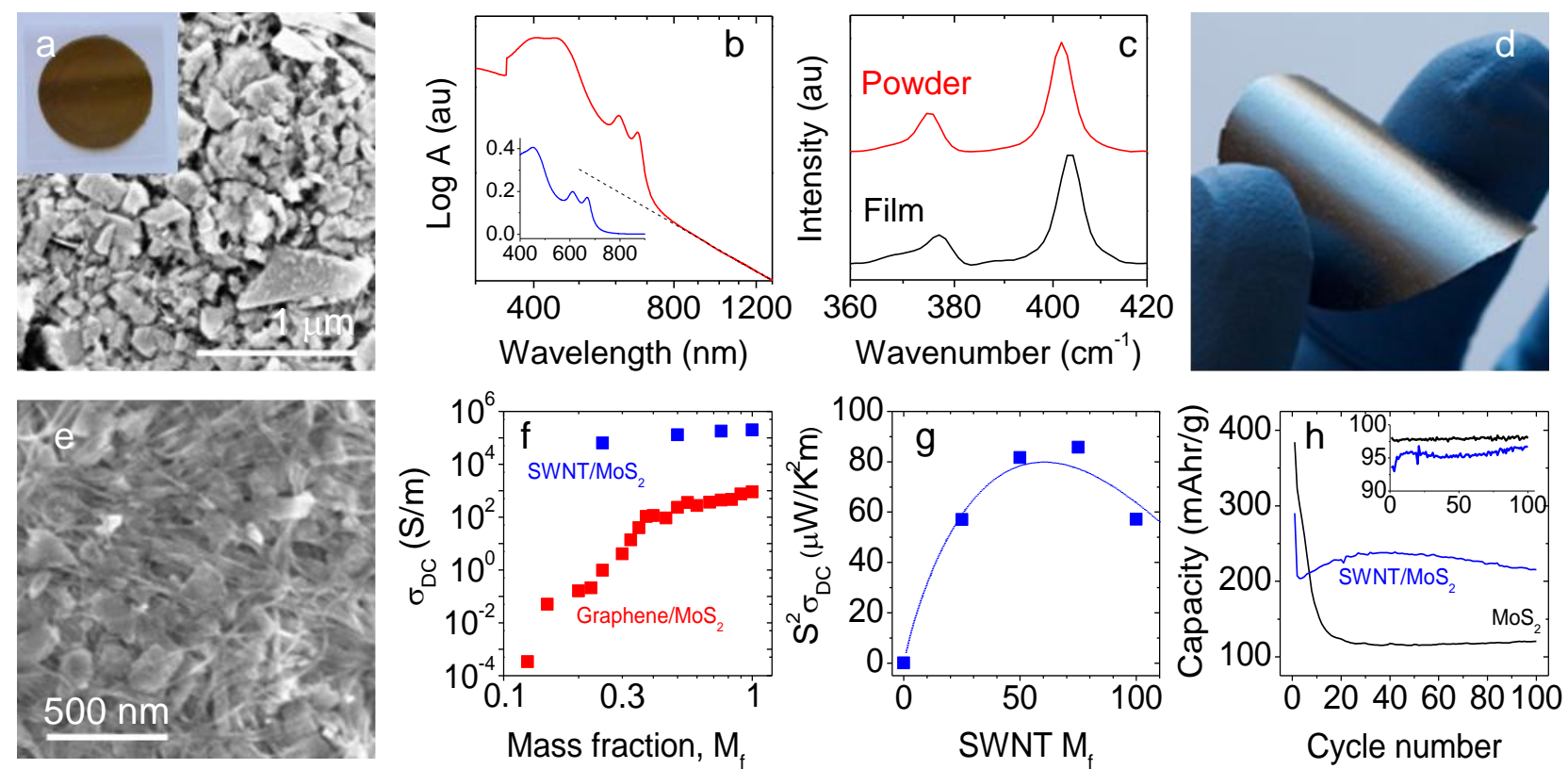

Figure 3: $\mathrm{MoS}_{2}$ based films and hybrids. a, Inset: A photograph of a thin (100s of nm) $\mathrm{MoS}_{2}$ film. Main image: An SEM image of the surface of a thin $\mathrm{MoS}_{2}$ film. b, An absorption spectrum of the film in a. Inset: The same absorption spectrum with the background (dashed line) subtracted. c, Raman spectra of both the film in a and the starting powder. $d$ and e, Photograph and SEM image of a thin $\mathrm{MoS}_{2} / \mathrm{SWNT}$ hybrid film. f, Electrical properties of $\mathrm{MoS}_{2} /$ graphene and $\mathrm{MoS}_{2} / \mathrm{SWNT}$ hybrid films as a function of mass fraction, $\mathrm{M}_{\mathrm{f}}$ (thickness $\sim 200 \mathrm{~nm}$ for $\mathrm{MoS}_{2} /$ graphene films and $\sim 50 \mu \mathrm{m}$ for $\mathrm{MoS}_{2} / \mathrm{SWNT}$ films). $\mathrm{g}$, Thermoelectric power factor, $\mathrm{S}^{2} \sigma_{\mathrm{DC}}$ (S is Seebeck coefficient) for $\mathrm{MoS}_{2} / \mathrm{SWNT}$ hybrid films as a function of SWNT $\mathrm{M}_{\mathrm{f}}$ (thickness $\sim 50 \mu \mathrm{m}$ ). The value for $\mathrm{MoS}_{2}$ was estimated from the literature as described in the SI. H, Lithium capacity as a function of charge/discharge cycle number for Li ion batteries with $\mathrm{MoS}_{2} / \mathrm{SWNT}$ and $\mathrm{MoS}_{2}$ films as the cathode. In each case the anode was lithium while the electrolyte was $\mathrm{LiPF}_{6}$ in ethylene carbonate/diethyl carbonate. Inset: Coulombic efficiency (\%) as a function of cycle number. 

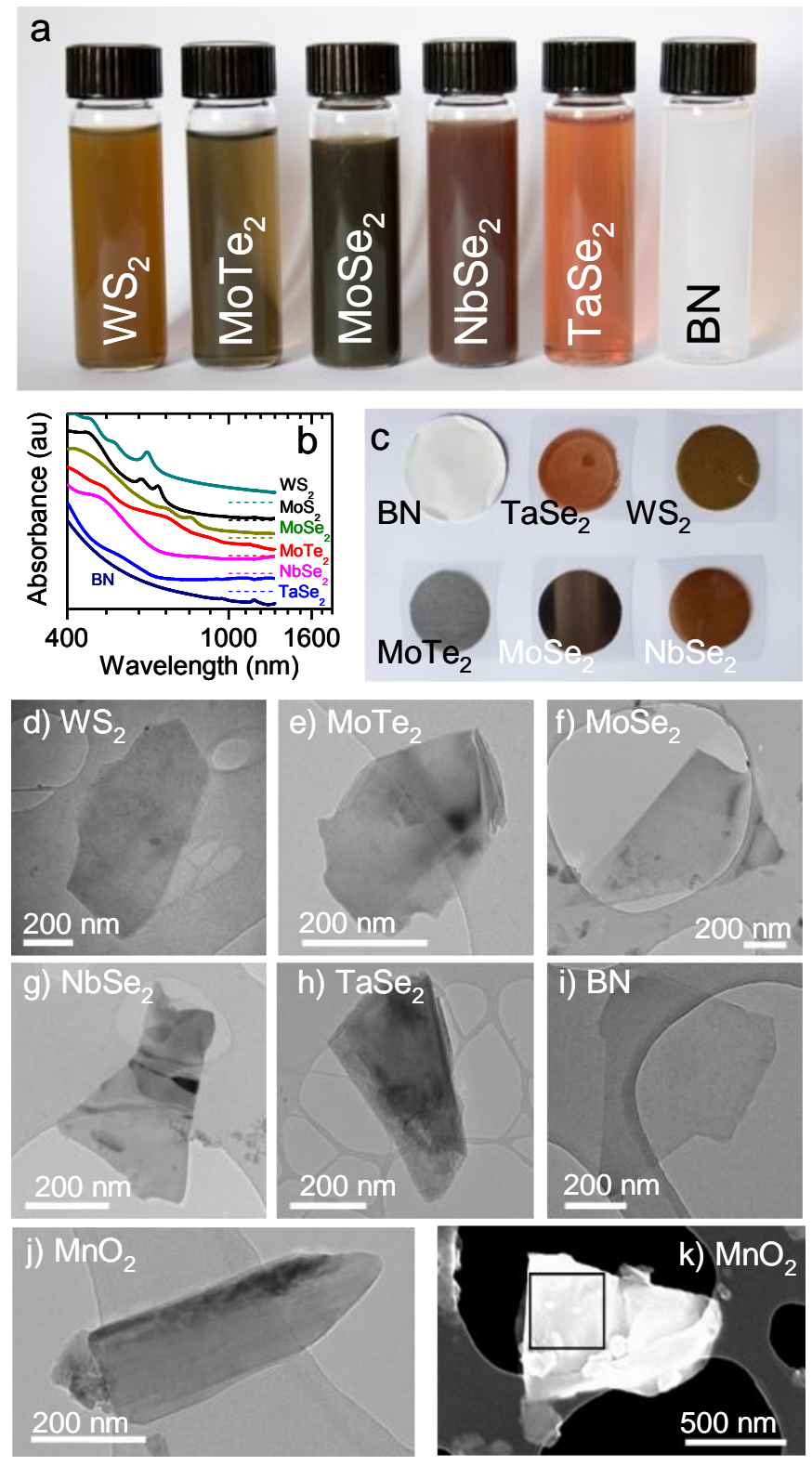

Figure 4: Dispersion of other inorganic layered compounds. a, Photograph of dispersions of $\mathrm{WS}_{2}$, $\mathrm{MoTe}_{2}, \mathrm{MoSe}_{2}, \mathrm{NbSe}_{2}, \mathrm{TaSe}_{2}$ and $\mathrm{BN}$ all stabilised in water by sodium cholate. $\mathrm{b}$, absorption spectra of dispersions shown in a. c, Vacuum filtered thin films of BN, TaSe $2, \mathrm{WS}_{2}, \mathrm{MoTe}_{2}, \mathrm{MoSe}_{2}$ and $\mathrm{NbSe}_{2}$ (note that due to its brittleness, the BN film is shown supported by a porous cellulose membrane) d-i, TEM images of flakes deposited on TEM grids from the dispersions in a. j, TEM image of an $\mathrm{MnO}_{2}$ flake stabilised in water using sodium cholate. Such flakes were both exfoliated from an $\mathrm{MnO}_{2}$ nanoparticulate powder where flakes were found as a minority phase. k) An SEM image of an $\mathrm{MnO}_{2}$ flake on a TEM grid. Energy dispersive X-ray spectral analysis, taken in the region marked by the box, confirmed the composition of this flake to be very close to $\mathrm{MnO}_{2}$. 